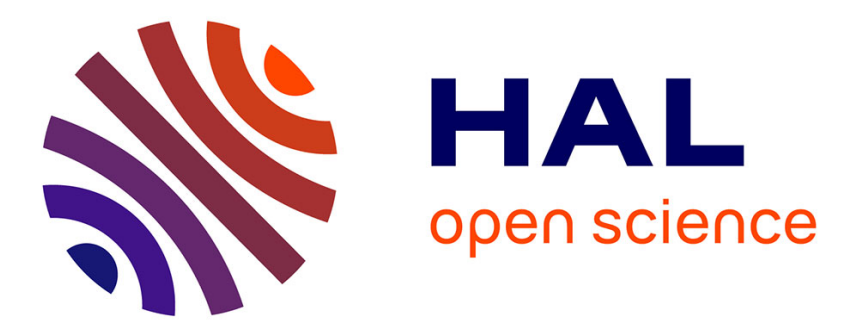

\title{
Shear Alignment of Bola-Amphiphilic Arginine-Coated Peptide Nanotubes
}

\author{
Ian W. Hamley, Samuel Burholt, Jessica Hutchinson, Valeria Castelletto, \\ Emerson Rodrigo da Silva, Wendel Alves, Philipp Gutfreund, Lionel Porcar, \\ Rajeev Dattani, Daniel Hermida-Merino, et al.
}

\section{To cite this version:}

Ian W. Hamley, Samuel Burholt, Jessica Hutchinson, Valeria Castelletto, Emerson Rodrigo da Silva, et al. Shear Alignment of Bola-Amphiphilic Arginine-Coated Peptide Nanotubes. Biomacromolecules, 2017, 18 (1), pp.141-149. 10.1021/acs.biomac.6b01425 . hal-01691803

\section{HAL Id: hal-01691803 https://hal.science/hal-01691803}

Submitted on 24 Jan 2018

HAL is a multi-disciplinary open access archive for the deposit and dissemination of scientific research documents, whether they are published or not. The documents may come from teaching and research institutions in France or abroad, or from public or private research centers.
L'archive ouverte pluridisciplinaire HAL, est destinée au dépôt et à la diffusion de documents scientifiques de niveau recherche, publiés ou non, émanant des établissements d'enseignement et de recherche français ou étrangers, des laboratoires publics ou privés. 


\section{Shear Alignment of Bola-Amphiphilic Arginine-Coated Peptide Nanotubes}

Ian W. Hamley,*0 Samuel Burholt, Jessica Hutchinson, and Valeria Castelletto

Department of Chemistry, University of Reading, Whiteknights, Reading, RG6 6AD, United Kingdom

Emerson Rodrigo da Silva

Instituto de Física, Universidade Federal de Alagoas, Maceió-Alabama 57072-900, Brazil

Wendel Alves ${ }^{\circ}$

Centro de Ciências Naturais e Humanas, Universidade Federal do ABC, Santo André 09210-580, Brazil

Philipp Gutfreund and Lionel Porcar

Institut Max von Laue - Paul Langevin, CS 20156; 71, Avenue des Martyrs F-38042 Grenoble Cedex 9, France

Rajeev Dattani, Daniel Hermida-Merino, and Gemma Newby

ESRF-The European Synchrotron, 38043 Grenoble Cedex 9, France

Mehedi Reza and Janne Ruokolainen

Department of Applied Physics, Aalto University School of Science, Aalto FI-00076, Finland

Joanna Stasiak

Department of Chemical Engineering \& Biotechnology, University of Cambridge, Pembroke Street, Cambridge, CB2 3RA, United Kingdom

S Supporting Information

ABSTRACT: The bola-amphiphilic arginine-capped peptide $\mathrm{RFL}_{4} \mathrm{RF}$ self-assembles into nanotubes in aqueous solution. The nanostructure and rheology are probed by in situ simultaneous rheology/small-angle scattering experiments including rheo-SAXS, rheo-SANS, and rheo-GISANS (SAXS: small-angle X-ray scattering, SANS: small-angle neutron scattering, GISANS: grazing incidence small-angle neutron scattering). Nematic alignment of peptide nanotubes under shear is observed at sufficiently high shear rates under steady shear in either Couette or cone-and-plate geometry. The extent of alignment increases with shear rate. A shear plateau is observed in a flow curve measured in the Couette geometry, indicating the presence of

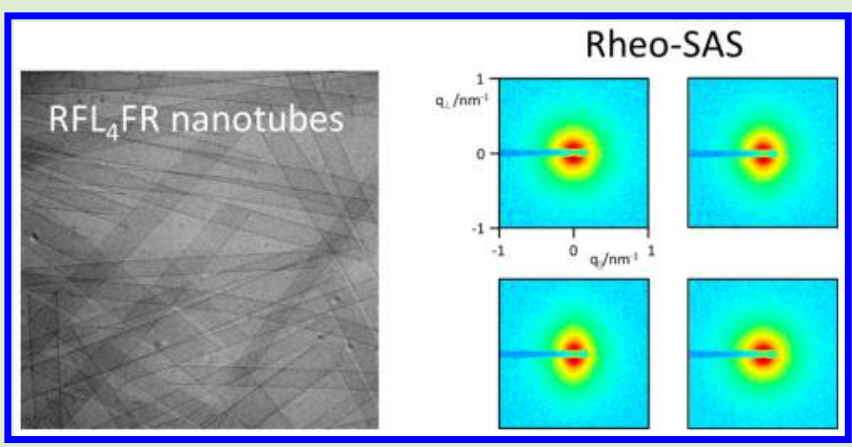
shear banding above the shear rate at which significant orientation is observed $\left(0.1-1 \mathrm{~s}^{-1}\right)$. The orientation under shear is transient and is lost as soon as shear is stopped. GISANS shows that alignment at the surface of a cone-and-plate cell develops at sufficiently high shear rates, very similar to that observed in the bulk using the Couette geometry. A small isotope effect (comparing $\mathrm{H}_{2} \mathrm{O} / \mathrm{D}_{2} \mathrm{O}$ solvents) is noted in the $\mathrm{CD}$ spectra indicating increased interpeptide hydrogen bonding in $\mathrm{D}_{2} \mathrm{O}$, although this does not influence nanotube self-assembly. These results provide new insights into the controlled alignment of peptide nanotubes for future applications.

\section{INTRODUCTION}

Peptide bola-amphiphiles are remarkable biocompatible molecules incorporating symmetric hydrophilic headgroups on each
Received: September 23, 2016

Revised: November 30, 2016

Published: December 1, 2016 
side of a central hydrophobic sequence. The aggregation properties of bola-amphiphilic peptides have recently been investigated by several groups. For example, the groups of Shimizu and Liu have observed nanotube formation by bola-amphiphiles comprising lengthy lipid central groups (e.g., $\mathrm{C}_{16}$ or $\mathrm{C}_{18}$ chains) group-capped with short oligopeptides. ${ }^{1-5} \mathrm{~A}$ recent paper reports a similar design (lipid-based central chains to which four dipeptides are attached in a $\mathrm{H}$-shaped molecule) as the basis of dipeptide bolaamphiphiles for siRNA delivery. ${ }^{6}$ Other groups have studied similar peptide end-capped bola-amphiphiles as discussed in a general review on bola-amphiphiles. ${ }^{7}$ In another recent example, it has been shown that the self-assembly of peptide $\mathrm{KI}_{4} \mathrm{~K}$ can be controlled by adjusting the solvent composition in water/acetonitrile mixtures-nanotubes, twisted ribbons, or fibrils being observed. ${ }^{8,9}$ By contrast, $\mathrm{KA}_{6} \mathrm{~K}$ was found not to self-assemble, this being ascribed to electrostatic repulsion of the lysine termini (the analogue $\mathrm{KA}_{6} \mathrm{E}$ however did form tapelike fibrils). ${ }^{10}$ Recently, twisted ribbons and fibrils (comprising bundled helical filaments) have been reported for $\mathrm{EF}_{4} \mathrm{E}$ and $\mathrm{KF}_{4} \mathrm{~K}$ respectively (mixtures self-assemble into tapes). ${ }^{11}$ The central block may comprise just hydrophobic residues as in the above-mentioned examples or for example alternating residues, giving rise to the so-called multidomain peptides studied by the Hartgerink group. ${ }^{12}$

In a recent study, we investigated in detail the self-assembly and secondary structure of the peptide bola-amphiphile $\mathrm{RFL}_{4} \mathrm{FR}^{13}$ This peptide comprises a hydrophobic tetra-leucine core to which terminal FR motifs are attached. These incorporate a cationic arginine residue to enable solubility in aqueous solutions and a phenylalanine residue to favor $\pi-\pi$ stacking interactions. This bola-amphiphile self-assembles into $\beta$-sheet structures above a critical aggregation concentration $(\mathrm{CAC}=$ 0.085 wt \%), which was determined using a pyrene fluorescent probe technique. The self-assembled morphology was shown to be nanosheets comprising stacked bilayers, as revealed by cryogenic transmission electron microscopy (cryo-TEM) and small-angle X-ray scattering (SAXS) and small-angle neutron scattering (SANS). The kinetics of aggregation were monitored by circular dichroism spectroscopy which indicated a nucleation process followed by an autocatalytic event. Films of the peptide were shown to be cytocompatible (using a human corneal stromal fibroblast model). In a related study, we examined the self-assembly of the complementary peptide $\mathrm{EFL}_{4} \mathrm{FE}$ with anionic glutamic acid terminal residues replacing the cationic arginine residues in $\mathrm{RFL}_{4} \mathrm{FR}^{14}$ This peptide self-assembles into nanotubes with slow kinetics ( $\sim$ days) as monitored by SAXS. $\mathrm{CD}$ measurements suggested, very unexpectedly, a loss of $\beta$-sheet structure over the same time scale, i.e., the final nanotube state comprises peptides in a largely unordered conformation. The peptide $\mathrm{RFL}_{4} \mathrm{FR}$ showed a highly aligned fiber X-ray diffraction pattern (from which a model for the peptide packing could be deduced), ${ }^{13}$ which motivated us to investigate alignment in further detail, under defined flow conditions. This is the subject of the present manuscript.

Here, the alignment of $\mathrm{RFL}_{4} \mathrm{FR}$ nanotubes under controlled steady shear conditions is investigated via a combination of grazing-incidence small-angle neutron scattering (GISANS) with simultaneous rheology and also bulk rheo-SANS (rheologySANS) and rheo-SAXS (rheology-SAXS). A detailed picture of flow alignment is obtained and this is correlated to the nonlinear flow behavior. In the present work, nanotube self-assembly was observed for $\mathrm{RFL}_{4} \mathrm{FR}$, i.e., the curved nanosheets previously observed $^{13}$ close into nanotubes after storage. This occurs over a time scale of weeks. Slow closure of peptide sheet/ribbon structures into nanotubes has been previously reported for other systems. $^{15,16}$

\section{EXPERIMENTAL SECTION}

Materials. Peptide $\mathrm{NH}_{2}$-RFLLLLFR-OH was custom synthesized by Biomatik (Ontario, Canada) and was received as the TFA salt variant. Two batches were received with purity respectively $93.6 \%$ and $96.3 \%$ by HPLC in water/acetonitrile containing 0.1\% TFA. Mass spectroscopy (ESI-MS) indicated a molar mass $1077.39 \mathrm{~g} \mathrm{~mol}^{-1}\left(1077.36 \mathrm{~g} \mathrm{~mol}^{-1}\right.$, expected). Samples were prepared by dissolving the peptide into $\mathrm{H}_{2} \mathrm{O}$ or $\mathrm{D}_{2} \mathrm{O}, 1$ or 3.5 wt $\%$ concentration, and storing at $4{ }^{\circ} \mathrm{C}$ for a period of weeks previous to further analyses.

Small-Angle X-ray Scattering (SAXS). SAXS data were obtained on beamline ID02 at the ESRF, Grenoble, France. Data were collected using a Pilatus $1 \mathrm{M}$ detector. Samples were placed into a Hake RS6000 stress-controlled rheometer with modified (polycarbonate) Couette cell of inner diameter $20 \mathrm{~mm}$ radius and $2 \mathrm{~mm}$ gap. Samples were subjected to shear at different shear rates in the range $\dot{\gamma}=0.1 \mathrm{~s}^{-1}$ to $1000 \mathrm{~s}^{-1}$. The sample-detector distance was either 1.2 or $10 \mathrm{~m}$. The X-ray wavelength ( $\lambda$ ) was $0.995 \AA$ and the wavenumber $q=4 \pi \sin \theta / \lambda$ (where $2 \theta$ is the scattering angle) scale was calibrated using silver behenate.

Small-Angle Neutron Scattering (SANS). SANS data were obtained on beamline D22 at the ILL, Grenoble, France. Solutions of $\mathrm{RFL}_{4} \mathrm{FR}$ were prepared using $\mathrm{D}_{2} \mathrm{O}$ as a solvent in order to minimize incoherent background scattering from the buffer. Samples were loaded into a stress-controlled rheometer from Anton Paar (Paar Physica MCR 501 ) with a custom-made quartz Couette geometry (30 mm diameter, $0.5 \mathrm{~mm}$ gap) that allows for simultaneous measurement of the rheological behavior and scattering patterns, termed rheo-SANS. Samples were subjected to shear at different shear rates in the range $\dot{\gamma}=0.1 \mathrm{~s}^{-1}$ to $1000 \mathrm{~s}^{-1}$. The sample-detector distance was $17 \mathrm{~m}, 5.6$ or $2.5 \mathrm{~m}$. Most data presented herein were obtained at $17 \mathrm{~m}$.

Grazing Incidence Small-Angle Neutron Scattering (GISANS). GISANS was performed on beamline FIGARO at the Institut Laue Langevin, Grenoble France. The measurements were performed in time-of-flight mode using a wavelength band from $2.2-16 \AA$ and a wavelength resolution of $7.4 \%$. The reflection angle was set to $0.62^{\circ}$ with a vertical and horizontal angular divergence of $0.022^{\circ}$ and $0.1^{\circ}$, respectively. The detector pixel resolution corresponds to an angular spread of $0.018^{\circ}$ and $0.16^{\circ}$, respectively. All resolutions are given as Gaussian equivalent full width at half-maximum (fwhm). The acquisition time was $5 \mathrm{~h}$ per pattern. The specularly reflected beam was masked during the data reduction.

The sample was contained in an Anton-Paar MCR 501 rheometer in cone/plate geometry ( $1^{\circ}$ cone angle, $50 \mathrm{~mm}$ cone diameter) to allow in situ rheology as explained in ref 17 . The neutrons pass the plate which is a single crystal silicon wafer polished to a roughness of $0.2 \mathrm{~nm}$ as determined by specular reflectivity measurements in pure water (NR, not shown). The surface was cleaned by ultrasonication in ethanol, acetone, toluene, and Millipore filtered water prior to the measurements. NR was also performed in between the GISANS measurements and no significant adsorption of peptide on the surface was detected during the experiment. Samples were subjected to shear at different shear rates in the range $=0.01 \mathrm{~s}^{-1}$ to $1000 \mathrm{~s}^{-1}$. The footprint of the neutron beam $\left(45 \times 11 \mathrm{~mm}^{2}\right)$ was centered to the cone, hence the scattering plane was parallel to the shear gradient and mainly shear flow plane; however, with some contributions of the vorticity direction close to the center of the cone. The temperature was kept constant at $20{ }^{\circ} \mathrm{C}$ throughout the GISANS experiment.

Cryogenic-Transmission Electron Microscopy (Cryo-TEM). Imaging was carried out using a field emission cryo-electron microscope (JEOL JEM-3200FSC), operating at $200 \mathrm{kV}$. Images were taken in bright field mode and using zero loss energy filtering (omega type) with a slit width of $20 \mathrm{eV}$. Micrographs were recorded using a Gatan Ultrascan $4000 \mathrm{CCD}$ camera. The specimen temperature was maintained at $-187^{\circ} \mathrm{C}$ during the imaging. Vitrified specimens were prepared using an automated FEI Vitrobot device using Quantifoil 3.5/1 holey carbon copper grids with a hole size of $3.5 \mu \mathrm{m}$. Just prior to use, 
grids were plasma cleaned using a Gatan Solarus 9500 plasma cleaner and then transferred into the environmental chamber of a FEI Vitrobot at room temperature and $100 \%$ humidity. Thereafter $3 \mathrm{~mL}$ of sample solution was applied on the grid and it was blotted twice for $5 \mathrm{~s}$ and then vitrified in a 1/1 mixture of liquid ethane and propane at temperature of $-180{ }^{\circ} \mathrm{C}$. The grids with vitrified sample solution were maintained at liquid nitrogen temperature and then cryo-transferred to the microscope.

Circular Dichroism (CD) Spectroscopy. CD spectra were recorded using a Chirascan spectropolarimeter (Applied Photophysics, UK). Samples of $\mathrm{RFL}_{4} \mathrm{FR}$ in $\mathrm{H}_{2} \mathrm{O}$ or $\mathrm{D}_{2} \mathrm{O}$ ( 1 wt \%), were placed in a sandwich cell $(0.01 \mathrm{~mm}$ spacing), spectra presented being with absorbance $A<2$ at any measured point with a $0.5 \mathrm{~nm}$ step, $1 \mathrm{~nm}$ bandwidth, and $1 \mathrm{~s}$ collection time per step. The $\mathrm{CD}$ signal from the background was subtracted from the $\mathrm{CD}$ data of the sample solutions.

\section{RESULTS}

Cryo-TEM images (Figure 1a,b) clearly show the formation of nanotubes in 1 wt \% solutions of $\mathrm{RFL}_{4} \mathrm{FR}$ after storage in the fridge for several weeks. Some "saddle-splay" curved nanosheets, forming mesh-like structures were observed in some areas of the TEM grids (Figure 1a,b and Figure S1), consistent with the formation of the nanotubes by wrapping of curved sheets. ${ }^{16}$ In the present study, samples were prepared in $\mathrm{D}_{2} \mathrm{O}$ for SANS measurements, in contrast to $\mathrm{H}_{2} \mathrm{O}$ used in a previous study. ${ }^{13}$ In order to check for any possible isotope effects on selfassembly, cryo-TEM images were also obtained in $\mathrm{H}_{2} \mathrm{O}$. These showed the same nanotube assemblies (Figure 1). The nanotube diameter was measured to be $(73.5 \pm 16.9) \mathrm{nm}$ from a series of images of samples ( $1 \mathrm{wt} \%$ ) in $\mathrm{H}_{2} \mathrm{O}$, the corresponding value for a sample in $\mathrm{D}_{2} \mathrm{O}$ being $(67.5 \pm 22.8) \mathrm{nm}$. There is no significant difference in values in the two solvents. Possible solvent isotope effects on peptide conformation were also examined by $C D$ spectroscopy. The CD spectra are presented in Figure S2 and, for the peptide in either $\mathrm{H}_{2} \mathrm{O}$ or $\mathrm{D}_{2} \mathrm{O}$, show maxima just above $200 \mathrm{~nm}$ and minima near $220 \mathrm{~nm}$, features consistent with $\beta$-sheet formation by extended peptide nanostructures. ${ }^{18}$
The spectrum observed in $\mathrm{D}_{2} \mathrm{O}$ does, however, show more intense maxima and minima, suggesting enhanced hydrogen bonding between peptides in $\mathrm{D}_{2} \mathrm{O}$, which is possibly an effect of competitive H-bonding peptide/peptide versus peptide/solvent. The formation of nanotubes in $1 \mathrm{wt} \%$ solutions of $\mathrm{RFL}_{4} \mathrm{FR}$ was also confirmed by modeling of SAXS and SANS data, as discussed below.

The formation of possible liquid crystal phases was examined. A polarized optical micrograph of a 1 wt $\% \mathrm{RFL}_{4} \mathrm{FR}$ solution in $\mathrm{H}_{2} \mathrm{O}$ shows isolated birefringent domains (Figure 1c) in aged solutions $\left(\sim 3\right.$ weeks, kept in the fridge at $\left.\sim 4{ }^{\circ} \mathrm{C}\right)$. However, upon shearing between a glass slide and coverslip nematic texture (Schlieren texture) is observed across the sample (Figure 1d). Fresh solutions at this concentration do not produce birefringence in the absence of shear. As mentioned above, aging is required in order to observe nanotube formation and at $1 \mathrm{wt} \%$ the nematic phase is induced by shear. The shear-induced alignment of the nematic phase was investigated by rheo-SANS and rheo-SAXS. Similar behavior was observed for a sample in $\mathrm{D}_{2} \mathrm{O}$, POM images before and after shearing for this sample being shown in Figure S3.

We used a combination of rheo-SANS and rheo-SAXS techniques to examine orientation. Bulk alignment effects in a Couette (concentric cylinder) were examined by rheo-SAXS and rheo-SANS, and structure-flow properties were investigated by simultaneous rheology/scattering measurements. This was contrasted with results from rheo-GISANS, which was used to probe alignment in a cone-plate geometry, probing orientation close to the plate wall. The shear geometries used are illustrated in Figure 2, which shows that for the bulk rheo-SAXS and rheoSANS measurements alignment was examined in two different planes with respect to the shear direction, so-called radial, and tangential configurations. This provides information on alignment in three dimensions.

Rheo-GISANS measurements were performed in order to correlate rheological behavior of the sample to structural

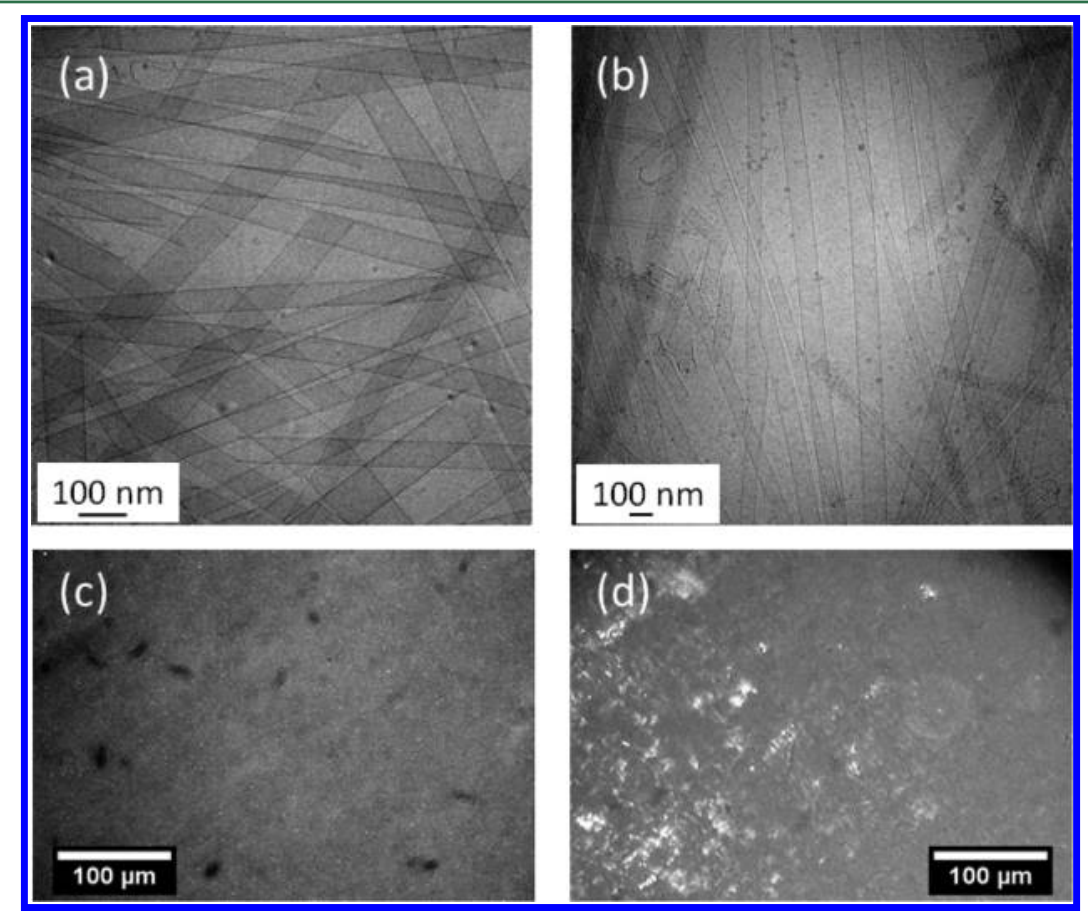

Figure 1. (a,b) Cryo-TEM images from 1 wt \% solutions of $\mathrm{RFL}_{4} \mathrm{FR}$ in $(\mathrm{a}) \mathrm{D}_{2} \mathrm{O}$, (b) $\mathrm{H}_{2} \mathrm{O}$. (c,d) Polarized optical micrographs of a 1 wt \% RFL $\mathrm{FR}$ solution in $\mathrm{H}_{2} \mathrm{O}$ between a glass slide and coverslip: (c) before shear, (d) after shear. 


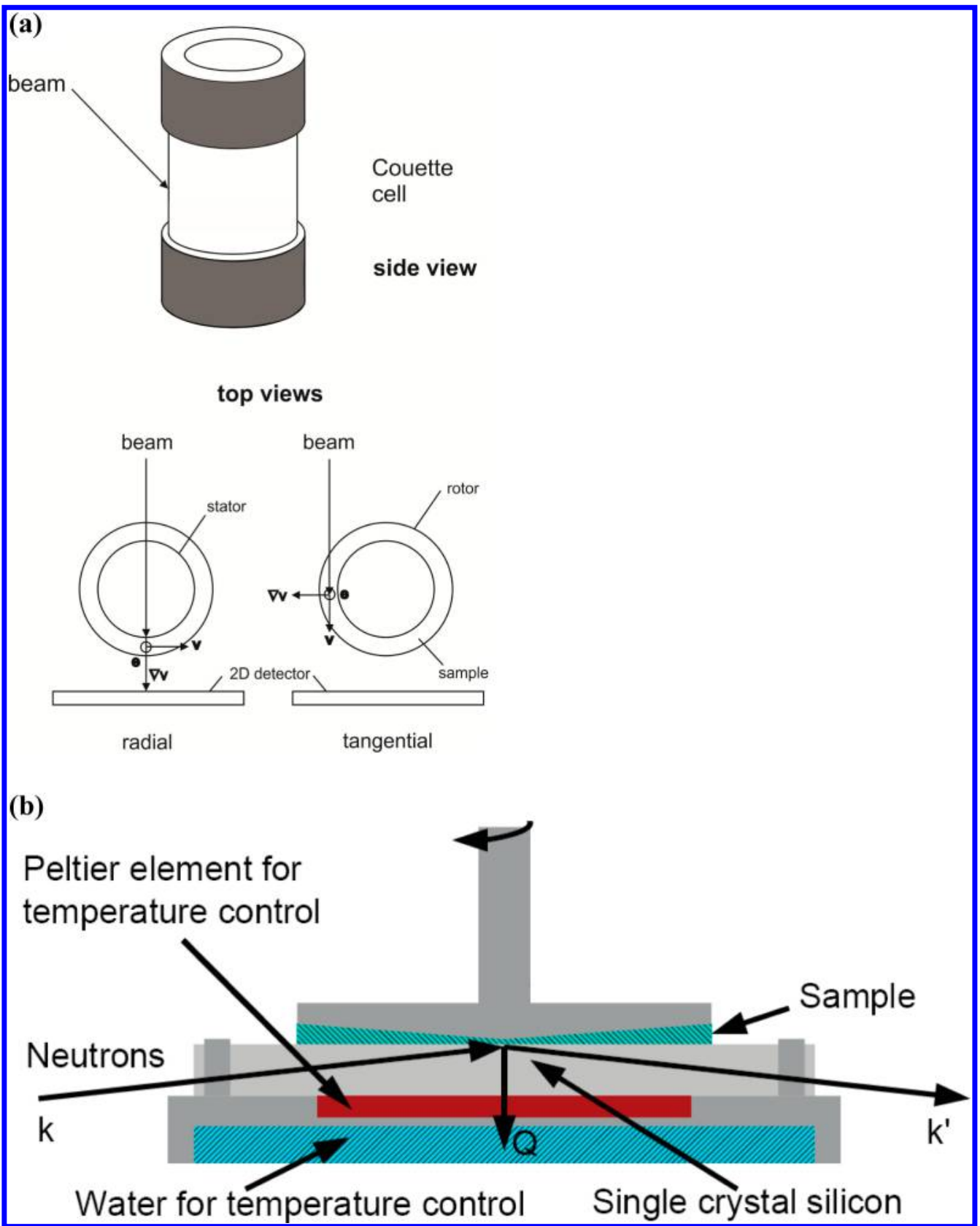

Figure 2. Definitions of geometry and shear axes. (a) SAXS/SANS, (b) GISANS.

alignment under flow close to the plate wall $(70 \mu \mathrm{m}$ penetration depth into the liquid). Grazing incidence scattering measurements provide a powerful tool to probe interfacial structure. ${ }^{19}$ Due to the glancing angle geometry most of the signal comes from a thin liquid layer close to the stationary plate. For the wavelength range used here, this layer thickness is on the order of several tens of micrometers. GISANS images obtained from a 1 wt \% solution of $\mathrm{RFL}_{4} \mathrm{FR}$ under the application of steady shear (and at rest) are shown in Figure 3. A slight degree of anisotropy becomes apparent at a shear rate of $1 \mathrm{~s}^{-1}$, but only at $100 \mathrm{~s}^{-1}$ and $1000 \mathrm{~s}^{-1}$ was pronounced anisotropy due to flow alignment noted. The anisotropy is in the form of prolate elliptical scattering contours, consistent with alignment of the peptide nanotubes along the flow direction (horizontal, QY). Due to the reflection geometry only about half of the scattering plane is accessible in GISANS as compared to SANS patterns. The rest is blocked by the sample horizon. The alignment is immediately lost following the cessation of shear as exemplified by the GISANS pattern in Figure 3a (obtained after shearing at $1000 \mathrm{~s}^{-1}$ ), which is isotropic. The corresponding bulk flow curves (viscosity vs shear rate) are shown in Figure 4 for two concentrations of the sample. The viscosity decreases dramatically with increasing shear rate above $\dot{\gamma}=10^{-3} \mathrm{~s}^{-1}$ indicating strong shear thinning behavior. However, as noted above, strong anisotropy in the GISANS patterns is not observed until much higher shear rates, i.e., in the strongly nonlinear flow regime. The flow curves also show an enhancement of the zero shear viscosity with increasing peptide concentration.

Rheo-SAXS was used to complement the rheo-GISANS, providing information on the alignment of the sample across the entire gap of the Couette call rather than only in the interfacial region of a cone/plate cell. SAXS data obtained from a 1 wt \% solution of $\mathrm{RFL}_{4} \mathrm{FR}$ under Couette flow in the radial geometry is shown in Figure 5. Significant alignment of the bulk sample was 
(a)

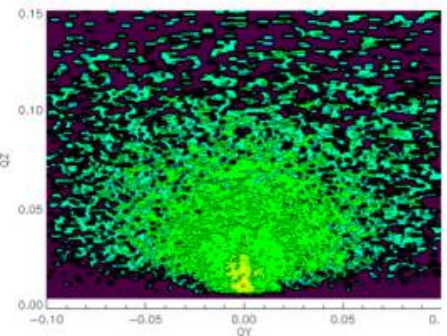

(d) (b)

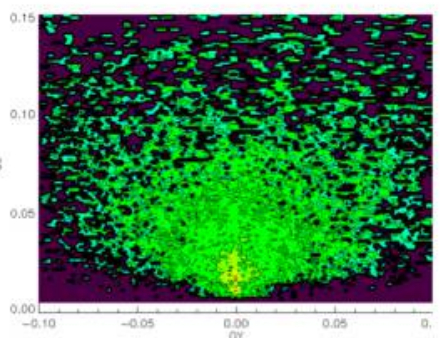

(c)

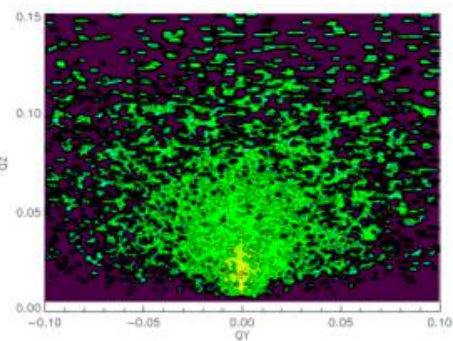

(e)
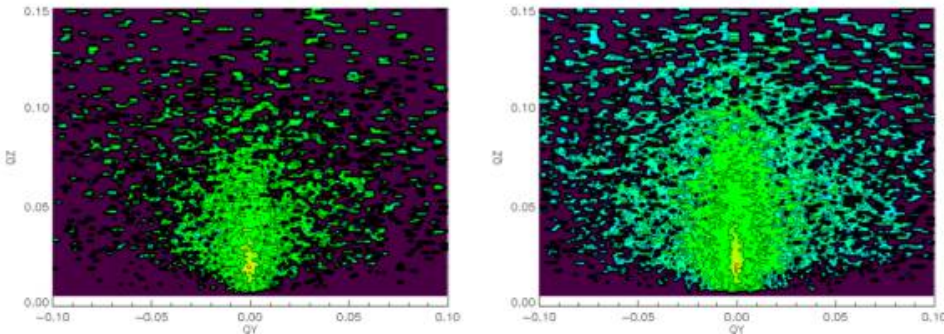

Figure 3. GISANS data from a $1 \mathrm{wt} \%$ solution of $\mathrm{RFL}_{4} \mathrm{FR}$ at rest or under steady shear (a) at rest (following shear at $\left.1000 \mathrm{~s}^{-1}\right),(\mathrm{b}) 0.01 \mathrm{~s}^{-1},(\mathrm{c}) 1 \mathrm{~s}^{-1}$, (d) $100 \mathrm{~s}^{-1}$, (e) $1000 \mathrm{~s}^{-1}$. The QZ axis is along the shear gradient, and the QY direction is mainly oriented along the shear flow. The axis units are in $\AA^{-1}$. The intensity is plotted on a logarithmic color scale.

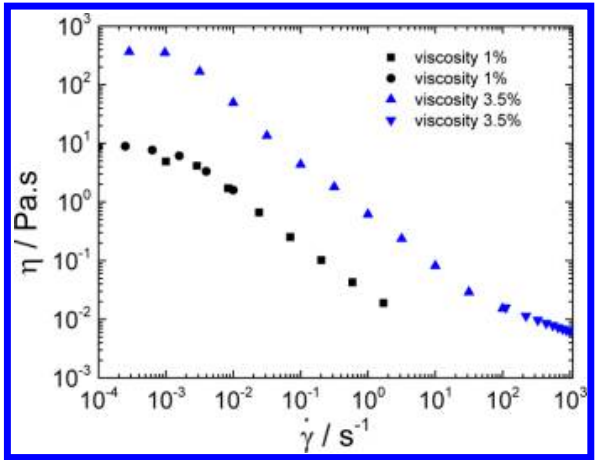

Figure 4. Steady state flow curves ( 1 and $3.5 \mathrm{wt} \% \mathrm{RFL}_{4} \mathrm{FR}$ ) in cone/plate geometry as used for the GISANS experiments. Flow curves ( 1 and 3.5 wt $\% \mathrm{RFL}_{4} \mathrm{FR}$ ) were measured for each sample over two ranges of shear rate (shown by different shaped symbols).

only observed while the sample was experiencing shear at higher shear rates at or above $\dot{\gamma}=1 \mathrm{~s}^{-1}$, no substantial orientation being observed in the SAXS pattern at lower shear rates studied (an example pattern for a sample under shear at $\dot{\gamma}=100 \mathrm{~s}^{-1}$ is shown in Figure $5 \mathrm{~b}$; data was also measured at $\dot{\gamma}=0.1,1$, and $10 \mathrm{~s}^{-1}$ ). The onset of significant alignment above $\dot{\gamma}=1 \mathrm{~s}^{-1}$ is in agreement with the GISANS results. Also of note, the anisotropy in the SAXS pattern was immediately lost following cessation of shear as shown in Figure 5d. This is due to the low viscosity of these samples (discussed quantitatively below), which means that shear-induced alignment at the macroscale is not retained in the absence of shear, although local alignment must be retained in the nematic phase. A previous study ${ }^{20}$ on peptide fibrils using SANS and rheology (performed separately) reported differences in response of fluid and gel nematic phases under shear-the fluid nematic phase showing no alignment in the absence of shear as in the present study, but in contrast to the nematic gel structure. More concentrated nematic phases (with higher viscosity due to fibril network formation) as for a PEG-peptide conjugate show retained nematic alignment after shearing. ${ }^{21,22}$ For $\mathrm{RFL}_{4} \mathrm{FR}$ solution,

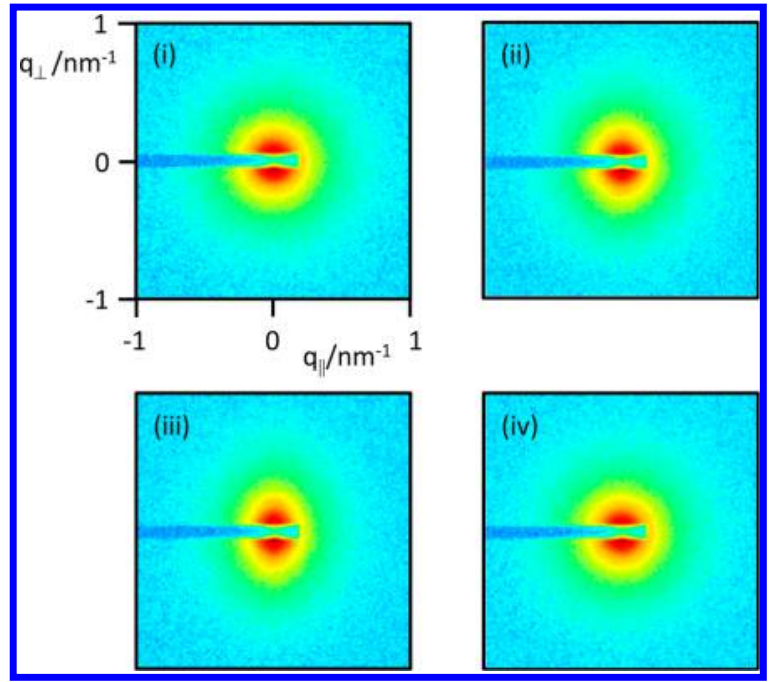

Figure 5. SAXS patterns obtained from a 1 wt $\%$ solution of $\mathrm{RFL}_{4} \mathrm{FR}$ in $\mathrm{D}_{2} \mathrm{O}$ in the radial configuration. (i) Zero shear, (ii) under shear at $\dot{\gamma}=100 \mathrm{~s}^{-1}$, (iii) under shear at $\dot{\gamma}=1000 \mathrm{~s}^{-1}$, (iv) following shear at $\dot{\gamma}=1000 \mathrm{~s}^{-1}$. The shear direction is horizontal and the intensity scale is logarithmic.

no orientation was observed in the tangential direction, even under shear at high shear rates (SAXS data not shown, SANS data discussed below). These patterns are consistent with alignment of the nanotubes in the $(\mathbf{v}, \nabla \mathbf{v})$ plane. Similar alignment was observed in the rheo-SANS measurements (also in a Couette geometry). Selected data are shown in Figure 6. Note that this data corresponds to lower $q$ and features from the form factor of nanotubes are visible, in particular the intensity maximum at $q=0.12 \mathrm{~nm}^{-1,}$ which is associated with the nanotube form factor as discussed shortly. The overall degree of alignment observed in the radial SANS patterns (Figure 6a) is similar to that in the SAXS measurements at the same shear rate. The same loss of alignment upon cessation of shear was noted. A set of radial SANS patterns at a full range of shear rates during and after shear are shown in Figure S4. 


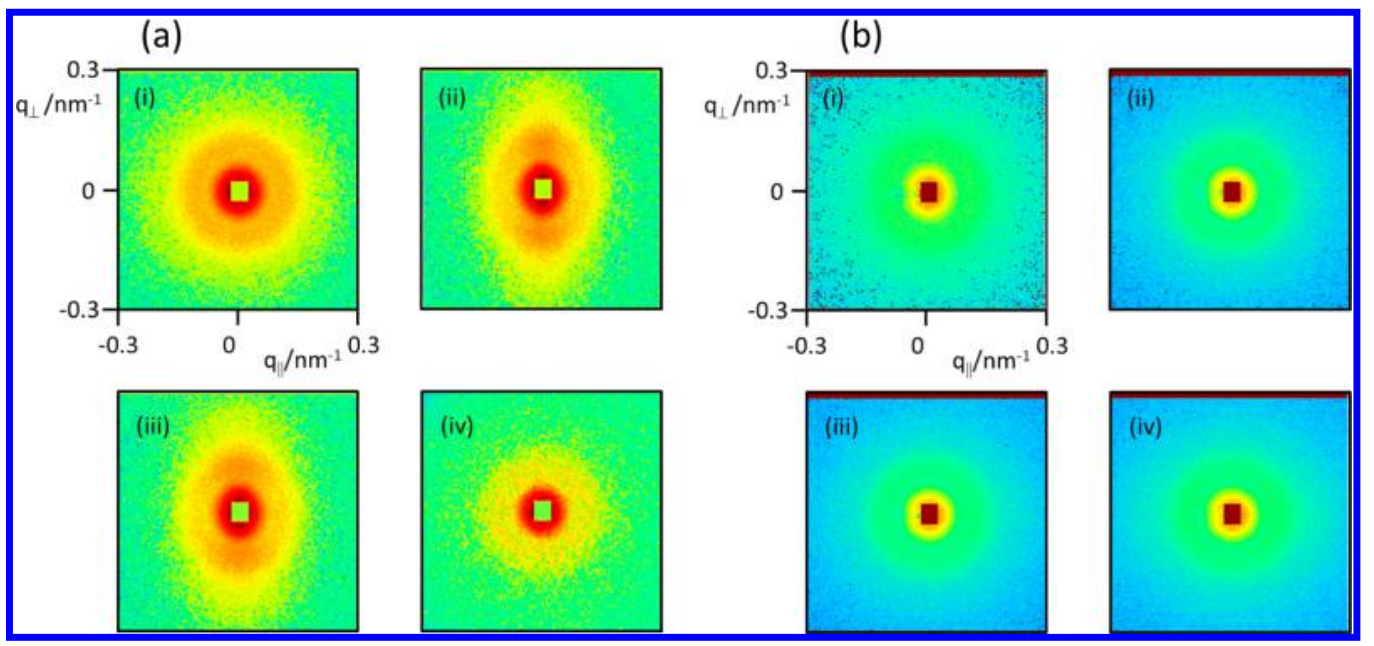

Figure 6. SANS patterns obtained from a 1 wt $\%$ solution of $\mathrm{RFL}_{4} \mathrm{FR}$ in $\mathrm{D}_{2} \mathrm{O}$ in (a) radial configuration. (i) Zero shear, (ii) under shear at $\dot{\gamma}=100 \mathrm{~s}^{-1}$ (iii) under shear at $\dot{\gamma}=1000 \mathrm{~s}^{-1}$, (iv) following shear at $\dot{\gamma}=1000 \mathrm{~s}^{-1}$. (b) Tangential SANS patterns. (i) Zero shear, (ii) under shear at $\dot{\gamma}=10 \mathrm{~s}^{-1}$, (iii) under shear at $\dot{\gamma}=100 \mathrm{~s}^{-1}$, (iv) under shear at $\dot{\gamma}=1000 \mathrm{~s}^{-1}$. The shear direction is horizontal and the intensity scale is logarithmic.

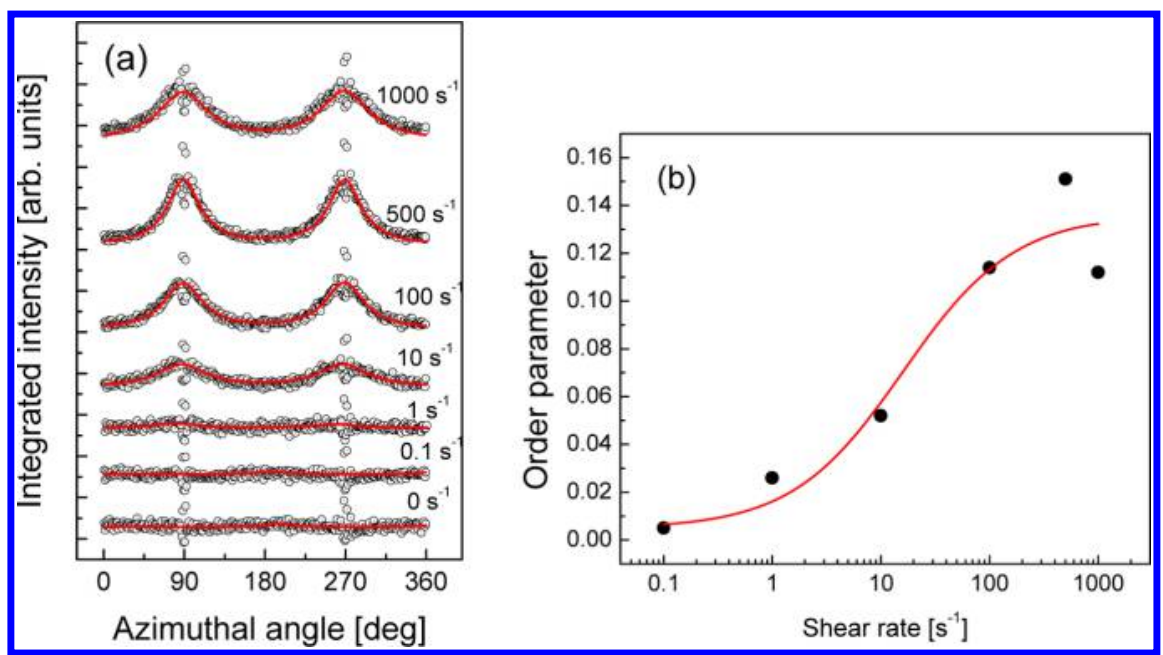

Figure 7. (a) Fitted azimuthal intensity profiles from integrated radial SANS patterns at the shear rates indicated. (b) Obtained order parameter $\bar{P}_{2}$ as a function of shear rate. The red line (logarithmic function) is a guide to the eye.

The degree of orientation in the SANS patterns in the radial configuration was quantified by fitting azimuthal intensity profiles (in a band centered on $q=0.12 \mathrm{~nm}^{-1}$ ) and extracting the orientational order parameter. The azimuthal intensity profiles as a function of azimuthal angle $\chi$ (defined with $\chi=0$ horizontal) were fitted to a double Lorentzian function: ${ }^{23}$

$$
I(\chi)=\frac{A}{w^{2}+\left(\chi-\chi_{0}\right)^{2}}+\frac{B}{w^{2}+\left(\chi-180-\chi_{0}\right)^{2}}+C
$$

Here $A$ and $B$ are fitted peak heights, and $C$ is a background term, $w$ sets the peak width, and $\chi_{0}$ the peak center. It should be noted that 2-fold symmetry in the anisotropic patterns (see Figure 6a) allows using peak centers shifted by $180^{\circ}$ and the same parameter $w$ for peak widths. Figure $7 \mathrm{a}$ shows fitted azimuthal intensity profiles.

The order parameter $\bar{P}_{2}=\left(3\left\langle\cos ^{2} \chi\right\rangle-1\right) / 2$ was calculated using the following average:

$$
\left\langle\cos ^{2} \chi\right\rangle=\frac{\int_{0}^{\pi} I(q, \chi) \cos ^{2} \chi \sin \chi \mathrm{d} \chi}{\int_{0}^{\pi} I(q, \chi) \sin \chi \mathrm{d} \chi}
$$

Figure $7 \mathrm{~b}$ shows the obtained order parameter as a function of shear rate. An S-shaped dependence on shear rate is noted. At the highest shear rate $\left(\dot{\gamma}=1000 \mathrm{~s}^{-1}\right)$, a small reduction in order parameter is observed, possibly due to sample being expelled at this high shear rate and/or bubble formation.

SANS patterns obtained in the tangential configuration (Figure 6b) show weak shear-rate dependent anisotropy (Figure S5 shows the corresponding azimuthal intensity profiles), and this is consistent with the cylindrical symmetry of the nanotubes viewed "end on" in the $(\nabla \mathbf{v}, \mathbf{e})$ plane. The residual anisotropy observed is due to the presence of a fraction of unwrapped nanotubes, i.e. nanosheets, in the sample. These align in the $(\mathbf{v}, \mathbf{e})$ plane producing horizontal Bragg peaks (azimuthal angle $0^{\circ}$ or $180^{\circ}$ ).

The form factor was obtained from 1D profiles of SAXS and SANS data obtained by integration of the $2 \mathrm{D}$ patterns. Data are shown in Figure 8a and a very similar form factor profile is observed in the SAXS and SANS data at low $q$ with a pronounced peak near $q=0.12 \mathrm{~nm}^{-1}$ plus higher order oscillations, particularly notable in the SAXS data. The combination of SAXS and SANS data span an exceptionally wide $q$ range (more than 2 orders of magnitude) enabling unprecedented insights 


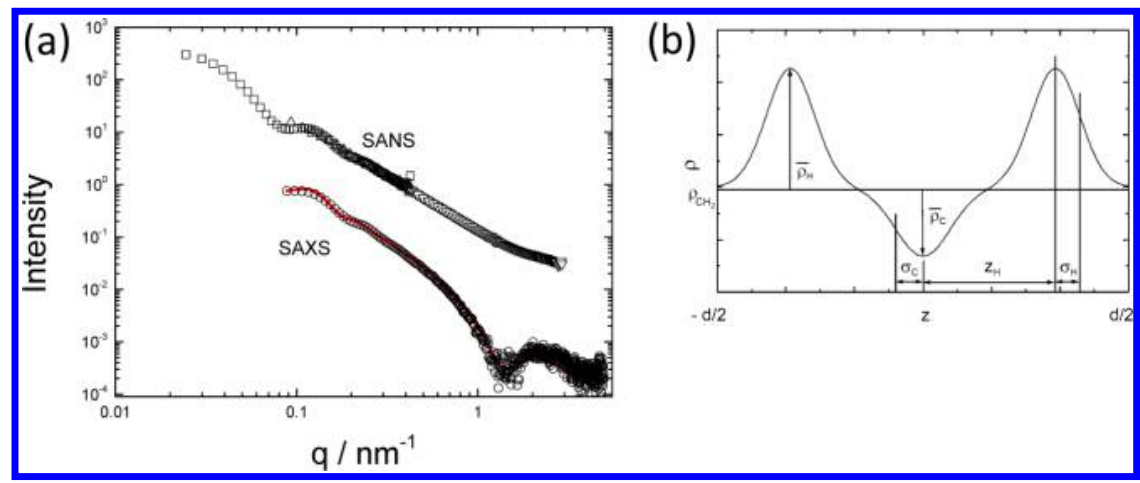

Figure 8. (a) One dimensional intensity profiles of SAXS and SANS data (open symbols) and form factor fitting (red line) of SAXS data to a nanotube + Gaussian bilayer model. The SANS data are combined from measurements at three sample-to-detector distances and presented as absolute intensities in $\mathrm{cm}^{-1}$. (b) Representation of electron density profile according to Gaussian bilayer model. ${ }^{25}$

into the nanotube structure. Since the SAXS data extends to higher $q$, it was used in form factor fits (performed using the software SASfit ${ }^{24}$ ). The data were fitted to a combination of two form factors of a hollow cylinder (to represent a nanotube structure) and a bilayer. We have successfully used this model to fit SAXS data from another lipopeptide nanotube system. ${ }^{16}$ The latter component represents the internal structure in the nanotube wall, represented (Figure $8 b$ ) as a sum of three Gaussians representing the electron density profile across the bilayer with electron dense regions at the two walls and electron poor regions in the lipid interior, based on a model for the lipid bilayer form factor. ${ }^{25}$ The fit parameters obtained indicate that the nanotube radius is $27.3 \mathrm{~nm}$ (5 nm Gaussian HWHM polydispersity) with a bilayer thickness (defined as the separation between the center of the two outer Gaussians $+2 \times$ Gaussian peak widths) of $3.2 \mathrm{~nm}$. These parameters are in good agreement with the cryo-TEM images (Figure 1) within uncertainties.

Rheology data obtained concurrently with the SAXS data is shown in Figure 9. The data is presented as time-dependent

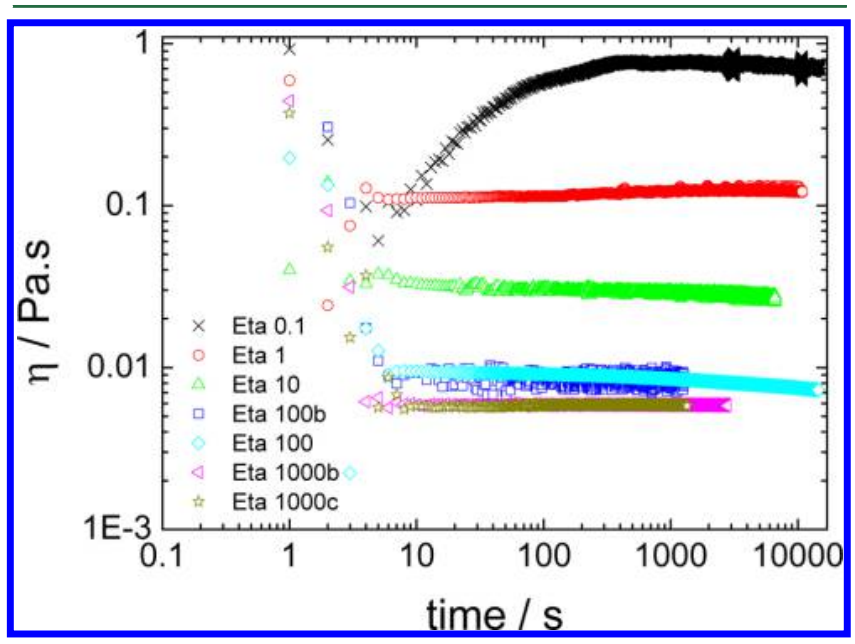

Figure 9. Time-dependent viscosity of a 1 wt \% solution of $\mathrm{RFL}_{4} \mathrm{FR}$ in $\mathrm{D}_{2} \mathrm{O}$ measured (concurrently with SAXS data) at different shear rates indicated. For convenience (in the representation of the data on a double logarithmic scale), the start of the experiment is set at $t=1 \mathrm{~s}$.

viscosity under steady shear. At all shear rates, except the lowest, an initial shear thinning behavior is observed in the first $10 \mathrm{~s}$ followed by the retention of a constant viscosity. The steady-state viscosity decreases with increased applied shear rate, consistent with shear thinning behavior. The data for $\dot{\gamma}=0.1 \mathrm{~s}^{-1}$ is anomalous, with an increase in viscosity after the initial decrease which is ascribed to initial sample inhomogeneity (this was the first shear experiment after the sample was loaded), that is only slowly eliminated under very slow shear mixing conditions. For reference, the viscosity of water is approximately $9 \times 10^{-4} \mathrm{~Pa} \cdot \mathrm{s}$ at $25^{\circ} \mathrm{C}$ and even at the highest shear rate, the steady-state viscosity of the sample is significantly higher due to the solution nanostructure. As shown by data from repeat measurements at higher shear rates (Figure 9), the results are highly reproducible. For comparison to the flow curve in Figure 4, obtained with the GISANS rheometer, a flow curve obtained using the in situ SAXS rheometer is shown in Figure 10. The decrease in viscosity

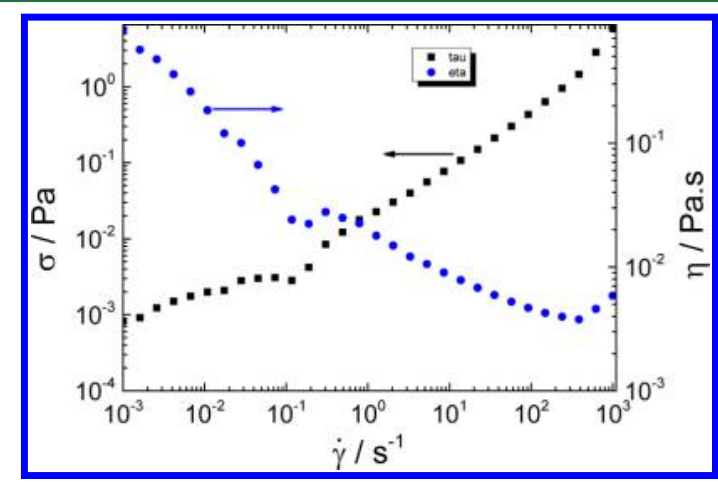

Figure 10. Flow curves in Couette geometry for rheo-SAXS for $1 \mathrm{wt} \%$ $\mathrm{RFL}_{4} \mathrm{FR}$ in $\mathrm{D}_{2} \mathrm{O}$.

with increasing shear rate indicates shear-thinning behavior; however, there is an apparent pseudoplateau region in the range $\dot{\gamma}=0.1-1 \mathrm{~s}^{-1}$. This behavior was reproducible in separate replicate off-line experiments performed in 1 wt $\% \mathrm{H}_{2} \mathrm{O}$ and $\mathrm{D}_{2} \mathrm{O}$ solutions. Plateaus in flow curves are signals for coexisting flow domains, so-called shear banding. ${ }^{26,29}$ The flow curves obtained are similar to those observed in the literature for wormlike micelle systems where a stress plateau is reached. ${ }^{28-30}$ However, since our peptide nanotube systems differ from wormlike micelles in many respects (persistence length, nature of the self-assembly process etc.) further comparison does not seem merited at this stage. It thus appears that under flow in a defined range of shear rates, in a 1 wt $\% \mathrm{RFL}_{4} \mathrm{FR}$ solution, there are coexisting bands which may have different degrees of alignment. In fact the shear rate $\dot{\gamma}=1 \mathrm{~s}^{-1}$ corresponds to the onset of slight anisotropy detected by SAXS so we believe that in the range $\dot{\gamma}=0.1-1 \mathrm{~s}^{-1}$ there may be partial alignment of the sample which is manifested under steady shear as one or more bands of aligned 
and unaligned sample. The data in Figure 10 is in agreement with the flow curve in cone-plate geometry obtained during GISANS measurements (Figure 4) at low shear rates but differs at higher shear rates, which is presumably due to the change of shear geometry and the absence of shear banding in the cone-plate geometry. The GISANS data allows us to infer that the observed alignment persists close to the stationary plate and thus pronounced shear banding can be excluded in the cone/plate geometry as this would lead to a significantly lower shear rate close to the plate contradicting the similar macroscopic shear rate dependence of the alignment in SANS and GISANS.

\section{CONCLUSIONS}

We have studied the shear-induced orientation of peptide nanotubes in the nematic phase. To our knowledge, we report the first observation of arginine-functionalized peptide nanotubes by self-assembly of a bola-amphiphilic peptide. The nanotubes are imaged by cryo-TEM and in situ dimensions are obtained by modeling small-angle scattering data, which spans more than 2 orders of magnitude in $q$ values providing parameters including the nanotube radius and those representing the detailed bilayer structure of the nanotube walls. The nanotubes are formed in either $\mathrm{H}_{2} \mathrm{O}$ or $\mathrm{D}_{2} \mathrm{O}$. The $\mathrm{H} / \mathrm{D}$ isotopic substitution in the solvent influences the $\mathrm{CD}$ spectra and increased peptide $\beta$-sheet formation is observed in $\mathrm{D}_{2} \mathrm{O}$, which is explained on the basis of to an increase in peptide-peptide H-bonding in preference to peptide-solvent H-bonding.

Shear alignment of peptide nanotubes leading to orientation of a nematic phase has not previously been examined as far as we are aware. We find that steady shear can be used to controllably orient the $\mathrm{RFL}_{4} \mathrm{FR}$ peptide nanotubes, the degree of orientation as characterized by the order parameter $\bar{P}_{2}$ increasing with shear rate. The alignment is not retained after shearing is stopped, which is presumably due to the low viscosity of the highly fluid sample (a more concentrated sample, 3 wt \% was also studied, but no alignment was observed). Strong shear thinning quantified during the simultaneous rheo-SAXS measurements in bulk is observed within the first $10 \mathrm{~s}$ of steady shearing. The flow curve in the Couette geometry exhibits a viscosity plateau in the range $0.1-1 \mathrm{~s}^{-1}$ corresponding to the onset of observed alignment in the SAXS patterns. The possible shear banding effect merits further study in future work (e.g., using optical imaging to resolve isotropic/anisotropic bands or spatially resolved SAXS to scan across the velocity gradient), and comparison to the large body of literature on this topic for wormlike micelle systems. The rheoGISANS measurements enable ordering near the surface in a cone-and-plate geometry to be compared to orientation in the bulk (probed in the Couette geometry with both rheo-SAXS and rheo-SANS measurements). The same alignment of the nanotubes under flow was observed, with significant alignment noted for similar shear rates to those for bulk orientation.

Arginine-coated peptide nanotubes may have applications in the fabrication of functional biomaterials, e.g., scaffolds for cell growth or extracellular matrix deposition and alignment may be important in such materials. Our study shows that controllable peptide nanotube orientation using shear flow is possible, and is switchable.

\section{ASSOCIATED CONTENT}

\section{S Supporting Information}

The Supporting Information is available free of charge on the ACS Publications website at DOI: 10.1021/acs.biomac.6b01425.
Additional cryo-TEM images, CD spectra, additional radial SANS patterns, azimuthal intensity profiles from tangential SANS patterns (PDF)

\section{AUTHOR INFORMATION} ORCID

Ian W. Hamley: 0000-0002-4549-0926

Wendel Alves: 0000-0002-8394-2751

\section{Notes}

The authors declare no competing financial interest.

\section{ACKNOWLEDGMENTS}

This work was supported by EPSRC Grant EP/L020599/to I.W.H. We thank the ESRF (ref SC-4267) and ILL for SANS (ref 9-13-674) and GISANS (beam time ref 8-02-782). We also thank E. Watkins for providing the algorithm for the TOF-GISANS data reduction. E.R.S. and W.A.A. acknowledge FAPESP for grants (2013/12674-6 and 2015/24018-1). A DOI (10.5291/ ILL-DATA.8-02-782) has now been minted for the ILL GISANS data: NEWBY GEMMA; DA SILVA Emerson Rodrigo; GUTFREUND Philipp; HAMLEY Ian; HERMIDA MERINO DANIEL and PEDERSEN Martin Nors. (2016). Shear-induced orientational order of the nematic phase of amyloid fibrils probed by in situ Rheo-GISANS. Institut Laue-Langevin (ILL) doi: 10.5291/ILL-DATA.8-02-782

\section{REFERENCES}

(1) Shimizu, T.; Kogiso, M.; Masuda, M. Vesicle Assembly in Microtubes. Nature 1996, 383, 487-488.

(2) Kogiso, M.; Ohnishi, S.; Yase, K.; Masuda, M.; Shimizu, T. Dicarboxylic Oligopeptide Bolaamphiphiles: Proton-Triggered SelfAssembly of Microtubes with Loose Solid Surfaces. Langmuir 1998, 14, 4978-4986.

(3) Zhan, C.; Gao, P.; Liu, M. Self-Assembled Helical SphericalNanotubes from an L-Glutamic Acid Based Bolaamphiphilic Low Molecular Mass Organogelator. Chem. Commun. 2005, 2005, 462-464.

(4) Gao, P.; Liu, M. Compression Induced Helical Nanotubes in a Spreading Film of a Bolaamphiphile at the Air/Water Interface. Langmuir 2006, 22, 6727-6729.

(5) Shen, Z.; Wang, T.; Liu, M. H-Bond and П-П Stacking Directed Self-Assembly of Two-Component Molecular Nanotubes: Tuning Length, Diameter and Wall Thickness. Chem. Commun. 2014, 50, 2096-2099.

(6) Eldredge, A. C.; Johnson, M. E.; Oldenhuis, N. J.; Guan, Z. Focused Library Approach to Discover Discrete Dipeptide Bolaamphiphiles for Sirna Delivery. Biomacromolecules 2016, 17, 3138-3144.

(7) Nuraje, N.; Bai, H. Y.; Su, K. Bolaamphiphilic Molecules: Assembly and Applications. Prog. Polvm. Sci. 2013, 38, 302-343.

(8) Zhao, Y. R.; Wang, J. Q.; Deng, L.; Zhou, P.; Wang, S. J.; Wang, Y. T.; Xu, H.; Lu, J. R. Tuning the Self-Assembly of Short Peptides Via Sequence Variations. Langmuir 2013, 29, 13457-13464.

(9) Zhao, Y. R.; Deng, L.; Wang, J. Q.; Xu, H.; Lu, J. R. Solvent Controlled Structural Transition of $\mathrm{KI}_{4} \mathrm{~K}$ Self-Assemblies: From Nanotubes to Nanofibrils. Lanomuir 2015, 31, 12975-12983.

(10) Castelletto, V.; Gouveia, R. J.; Connon, C. J.; Hamley, I. W.; Seitsonen, J.; Nykänen, A.; Ruokolainen, J. Alanine-Rich Amphiphilic Peptide Containing the Rgd Cell Adhesion Motif: A Coating Material for Human Fibroblast Attachment and Culture. Biomater. Sci. 2014, 2, $362-369$.

(11) Hu, Y.; Lin, R.; Zhang, P. C.; Fern, J.; Cheetham, A. G.; Patel, K.; Schulman, R.; Kan, C. Y.; Cui, H. G. Electrostatic-Driven Lamination and Untwisting of Beta-Sheet Assemblies. ACS Nano 2016, 10, 880888.

(12) Dong, H.; Paramonov, S. E.; Aulisa, L.; Bakota, E. L.; Hartgerink, J. D. Self-Assembly of Multidomain Peptides: Balancing Molecular 
Frustration Controls Conformation and Nanostructure. $\underline{\text { L. Am. Chem. }}$ Soc. 2007, 129, 12468-12472.

(13) da Silva, E. R.; Walter, M. N. M.; Reza, M.; Castelletto, V.; Ruokolainen, J.; Connon, C. J.; Alves, W. A.; Hamley, I. W. SelfAssembled Arginine-Capped Peptide Bolaamphiphile Nanosheets for Cell Culture and Controlled Wettability Surfaces. Biomacromolecules 2015, 16, 3180-3190.

(14) da Silva, E. R.; Alves, W. A.; Castelletto, V.; Reza, M.; Ruokolainen, J.; Hussain, R.; Hamley, I. W. Self-Assembly Pathway of Peptide Nanotubes Formed by a Glutamatic Acid-Based Bolaamphiphile. Chem. Commun. 2015, 51, 11634-11637.

(15) Adamcik, J.; Castelletto, V.; Hamley, I. W.; Mezzenga, R. Direct Observation of Time-Resolved Polymorphic States in Self-Assembly of End-Capped Heptapeptides. Angew. Chem. Int. Ed. 2011, 50, 54955498.

(16) Hamley, I. W.; Dehsorkhi, A.; Castelletto, V.; Furzeland, S.; Atkins, D.; Seitsonen, J.; Ruokolainen, J. Reversible Helical Ribbon Unwinding Transition of a Self-Assembling Peptide Amphiphile. Soft Matter 2013, 9, 9290-9293.

(17) Wolff, M.; Kuhns, P.; Liesche, G.; Ankner, J. F.; Browning, J. F.; Gutfreund, P. Combined Neutron Reflectometry and Rheology. I. Appl. Crvstallogr. 2013, 46, 1729-1733.

(18) Hamley, I. W.; Nutt, D. R.; Brown, G. D.; Miravet, J. F.; Escuder, B.; Rodríguez-Llansola, F. Influence of the Solvent on the Self-Assembly of a Modified Amyloid Beta Peptide Fragment. Ii. Nmr and Computer Simulation Investigation. I. Phvs. Chem. B 2010, 114, 940-951.

(19) Hexemer, A.; Müller-Buschbaum, P. Advanced Grazing-Incidence Techniques for Modern Soft-Matter Materials Analysis. IUCrI 2015, 2, $106-125$.

(20) Mawer, P. J.; Waigh, T. A.; Harding, R.; McLeish, T. C. B.; King, S. M.; Bell, M.; Boden, N. Small-Angle Neutron Scattering from Peptide Nematic Fluids and Hydrogels under Shear. Langmuir 2003, 19, 49404949.

(21) Hamley, I. W.; Krysmann, M. J.; Castelletto, V.; Kelarakis, A.; Noirez, L.; Hule, R. A.; Pochan, D. Nematic and Columnar Ordering of a Peg-Peptide Conjugate in Aqueous Solution. Chem. - Eur. J. 2008, 14, 11369-11374.

(22) Hamley, I. W.; Krysmann, M. J.; Newby, G. E.; Castelletto, V.; Noirez, L. Orientational Ordering in the Nematic Phase of a PegPeptide Conjugate in Aqueous Solution. Phvs. Rev.E 2008, 77, 062901.

(23) Silva, B. F. B.; Zepeda-Rosales, M.; Venkateswaran, N.; Fletcher, B. J.; Carter, L. G.; Matsui, T.; Weiss, T. M.; Han, J.; Li, Y. L.; Olsson, U.; Safinya, C. R. Nematic Director Reorientation at Solid and Liquid Interfaces under Flow: Saxs Studies in a Microfluidic Device. Langmuir 2015, 31, 4361-4371.

(24) Bressler, I.; Kohlbrecher, J.; Thünemann, A. F. Sasfit: A Tool for Small-Angle Scattering Data Analysis Using a Library of Analytical Expressions. I. Appl. Crvstallogr. 2015, 48, 1587-1598.

(25) Pabst, G.; Rappolt, M.; Amenitsch, H.; Laggner, P. Structural Information from Multilamellar Liposomes at Full Hydration: Full QRange Fitting with High Quality X-Ray Data. Phvs. Rev. E: Stat. Phvs., Plasmas, Fluids. Relat. Interdiscip. Top. 2000, 62, 4000-4009.

(26) Berret, J.-F.; Roux, D. C.; Porte, G. Isotropic-to-Nematic Transition in Wormlike Micelles under Shear. I. Phvs. II 1994, 4, 1261-1279.

(27) Holmqvist, P.; Daniel, C.; Hamley, I.; Mingvanish, W.; Booth, C. Inhomogeneous Flow in a Micellar Solution of a Diblock Copolymer: Creep Rheometry Experiments. Colloids Surf. A 2002, 196, 39-50.

(28) Vermant, J. Large-Scale Structures in Sheared Colloidal Dispersions. Curr. Opin. Colloid Interface Sci. 2001, 6, 489-495.

(29) Liberatore, M. W.; Nettesheim, F.; Wagner, N. J.; Porcar, L. Spatially Resolved Small-Angle Neutron Scattering in the 1-2 Plane: A Study of Shear-Induced Phase-Separating Wormlike Micelles. Phvs. Rev. E 2006, 73, 020504.

(30) Becu, L.; Anache, D.; Manneville, S.; Colin, A. Evidence for Three-Dimensional Unstable Flows in Shear-Banding Wormlike Micelles. Phys. Rev. E 2007, 76, 011503. 